\title{
Asociación entre obesidad y tasa de filtración glomerular en población con hipertensión arterial
}

\author{
Nhoreidy Bautista-Florez ${ }^{1}$, Karla María Vega-González ${ }^{2}$, Aida Nery Figueroa-Cabrera ${ }^{1}$, Jorge A Ramos-Castaneda ${ }^{1}$ \\ ${ }^{1}$ Universidad Surcolombiana. Neiva. Colombia \\ ${ }^{2}$ Empresa Social del Estado Carmen Emilia Ospina. Neiva. Colombia
}

Como citar este artículo:

Bautista-Florez N, Vega-González CM, Figueroa-Cabrera AN, Ramos-Castaneda JA. Asociación entre obesidad y tasa de filtración glomerular en población con hipertensión arterial.

Enferm Nefrol. 2021 Ene-Mar;24(1):47-54

\section{Resumen}

Introducción: La obesidad tiene consecuencias para el sistema de salud por su asociación con la morbimortalidad y la afectación de la calidad de vida. A nivel individual genera proteinuria y glomeruloesclerosis, que aceleran la disminución de la filtración glomerular.

Objetivo: Determinar la asociación entre obesidad y tasa de filtración glomerular en población con hipertensión arterial.

Material y método: Estudio descriptivo de corte transversal, que analizó la obesidad y filtración glomerular en 3.197 pacientes con hipertensión arterial de una institución de primer nivel de atención. Se revisaron historias clínicas obteniendo variables sociodemográficas, antropométricas, parámetros clínicos, y clasificación de la enfermedad renal crónica.

Resultados: La población tuvo un promedio de edad de $64 \pm 12,47$ años, y en su mayoría fueron del sexo femenino $(70,58 \%)$. La prevalencia de obesidad grado I fue $(26,11 \%)$, grado II $(9,63 \%)$ y grado III $(4,56 \%)$. Los obesos tuvieron una mediana de la tasa de filtrado glomerular superior comparado con los no obesos, pero

\section{Correspondencia:}

Jorge A Ramos-Castaneda

Email: andres.ramos@usco.edu.co dicha diferencia solo fue observada en los estadios de ERC I y II.

Conclusión: Existe asociación entre obesidad e incremento de la tasa de filtrado glomerular solamente en estadios I y II. El perímetro abdominal, la tensión arterial no controlada y el LDL fueron variables que influyen en el promedio de la tasa de filtrado glomerular, pero tienen un comportamiento diferente por grado de enfermedad renal crónica.

PALABRAS CLAVE: enfermedad renal; obesidad; tasa de filtración glomerular; hipertensión.

\section{Association between obesity and glomerular filtration rate in a population with arterial hypertension}

\footnotetext{
Abstract

Introduction: Obesity has consequences for the health system due to the association with morbidity and mortality and the effect on quality of life. At the individual level, obesity generates proteinuria and glomerulosclerosis that accelerate the decrease in glomerular filtration.

Objective: To determine the association between obesity and glomerular filtration rate in a population with arterial hypertension.
} 
Methodology: A descriptive cross-sectional study that analyzed obesity and glomerular filtration in 3,197 patients with arterial hypertension from a first-level care institution. Medical records were reviewed obtaining sociodemographic and anthropometric variables, clinical parameters, and classification of chronic kidney disease.

Results: The population had an average age of 64 years (SD 12.47), and the majority were female $(70.58 \%)$. The prevalence of obesity grade I was $(26.11 \%)$, grade II $(9.63 \%)$ and grade III (4.56\%). Obese had a median rate of glomerular filtration superior compared to nonobese, but this difference was observed only in stages I and grade II grade.

Conclusion: There is an association between obesity and an increase in the glomerular filtration rate only in stages I and II. Abdominal circumference, uncontrolled blood pressure and LDL were variables that influence the average glomerular filtration rate, having a different behavior by degree of chronic kidney disease.

KEYWORDS: kidney disease; obesity; glomerular filtration rate; hypertension.

\section{Introducción}

La obesidad es una enfermedad crónica, caracterizada por una acumulación anormal o excesiva de grasa ${ }^{1}, y$ es considerada un problema de salud pública por las consecuencias que genera para el sistema de salud, por su asociación con la morbimortalidad del paciente y la afectación de la calidad de vida². La causa fundamental de la obesidad es un desequilibrio energético entre calorías consumidas y calorías gastadas, destacando una ingesta de alimentos ricos en grasa, sal y azúcares, pero deficientes en vitaminas, minerales y otros micronutrientes ${ }^{3,4}$. Otro factor desencadenante es la disminución de la actividad física producto del estilo de vida sedentario, mayor sistematización de las actividades laborales y los métodos modernos de transporte ${ }^{5,6}$.

El aumento del sobrepeso y la obesidad en la población adulta permite proyectar un incremento en la morbimortalidad en los próximos años ${ }^{7,8}$. Según la Organización Mundial de la Salud (OMS) en el año 2016 el $13 \%$ de la población adulta mundial (un $11 \%$ de los hombres y un $15 \%$ de las mujeres) eran obesos ${ }^{3}$. En Europa la prevalencia de la obesidad ha aumenta- do considerablemente 9 . Se estima que algunos países europeos, como España, el $17 \%$ y $37 \%$ de la población tenga obesidad y sobrepeso respectivamente ${ }^{5}$. En México alrededor del $32,8 \%$ de la población es obesa y en Colombia el $56,5 \%$ de la población mayor de 18 años presenta sobrepeso u obesidad ${ }^{10}$.

La obesidad se clasifica dentro de las enfermedades no transmisibles, y su principal problema son las consecuencias que genera para la salud, debido a que es un factor de riesgo para enfermedades cardiovasculares, accidente vascular cerebral isquémico, diabetes mellitus tipo 2 (DM II), osteoartritis, enfermedad vesicular, hipertensión arterial (HTA) y algunos cánceres (incluyendo cáncer de mama y de colon) 3,11,12. Además, es una causa reconocida de proteinuria y de glomeruloesclerosis segmentaria y focal, que acelera la progresión de cualquier tipo de nefropatía ${ }^{13,14}$. Así mismo, la glomerulopatía relacionada con la obesidad se caracteriza por el aumento de volumen del glomérulo, ligera hipercelularidad, engrosamiento de la membrana basal glomerular, teniendo como resultado una disminución lenta y progresiva de la tasa de filtración glomerular (TFG) ${ }^{15}$.

Algunos estudios han demostrado la asociación entre obesidad y la disminución de la TFG, encontrando que la obesidad puede provocar un síndrome de hiperfiltración glomerular, lo que explicaría la existencia de microalbuminuria, albuminuria, y en pacientes hospitalizados mayor frecuencia y severidad de daño renal agudo ${ }^{14,16-19}$. De igual manera, la obesidad y su impacto sobre la TFG ha sido estudiada en pacientes diabéti$\cos ^{20}$, pero aún se desconoce su asociación en pacientes con HTA. Por lo anterior, el objetivo del estudio fue determinar la asociación entre obesidad y TFG en población con HTA en una institución de primer nivel de atención ambulatoria.

\section{Material y Método}

Estudio descriptivo de corte transversal, con enfoque analítico, que evaluó la asociación entre la obesidad y la TFG en una población con HTA de una institución de primer nivel de atención en una ciudad al sur de Colombia.

La población estuvo conformada por personas adultas con diagnóstico de HTA registrados en el programa de riesgo cardiovascular de la institución de primer nivel de atención. La muestra fueron 3.197 pacientes registrados en la base de datos en la consulta del pro- 
grama durante el 2019. Se incluyeron los pacientes con diagnóstico médico de HTA, y que tuvieran registro de perfil lipídico, peso, talla, perímetro abdominal, creatinina y albuminuria. Se excluyeron personas con patologías como: DM, lupus, enfermedad renal aguda, reflujo vesico-ureteral, litiasis renal y glomerulonefritis.

Se utilizó un cuestionario dirigido, elaborado por los investigadores y validado por médicos y enfermeras especialistas en Nefrología. Se realizó una prueba piloto para determinar la capacidad de procesamiento de las variables y reducción de errores sistemáticos en el manejo de los datos. Se revisaron las historias clínicas de los pacientes recogiendo variables sociodemográficas (edad, sexo), presión arterial, parámetros analíticos (albuminuria, perfil lipídico), variables antropométricas (peso, talla, perímetro abdominal, clasificación sobrepeso y obesidad según índice de masa corporal) y clasificación de la enfermedad renal crónica (ERC) según TFG de Cockcroft-Gault ${ }^{20}$.

Algunas variables fueron categorizadas de la siguiente manera; tensión arterial no controlada mayor o igual a $135 / 85 \mathrm{mmHg}$, perímetro abdominal en riesgo mayor de $90 \mathrm{~cm}$ en hombres y $80 \mathrm{~cm}$ en mujeres. Los parámetros clínicos alterados fueron: colesterol total mayor de 200 $\mathrm{mg} / \mathrm{dl}, \mathrm{HDL}$ menor de $35 \mathrm{mg} / \mathrm{dl}$ en hombres y $45 \mathrm{mg} /$ dl en mujeres, LDL mayor a $160 \mathrm{mg} / \mathrm{dl}$ y triglicéridos mayor de $150 \mathrm{mg} / \mathrm{dl}$. La creatinina alterada fue aquella con valores por encima de $1,1 \mathrm{mg} / \mathrm{dl}$ en mujeres y 1,3 $\mathrm{mg} / \mathrm{dl}$ en hombres, y la microalbuminuria se definió entre $30-300 \mathrm{mg} / 24 \mathrm{~h}$.

Los criterios diagnósticos de ERC fueron los denominados marcadores de daño renal (kidney damage) o la reducción del filtrado glomerular $(\mathrm{FG})$ por debajo de 60 $\mathrm{ml} / \mathrm{min} / 1,73 \mathrm{~m}^{21}$. Tras la confirmación diagnóstica, la ERC se clasificó según las categorías de FG, albuminuria y según la etiología 22 .

El sesgo de información se controló con la medición de la variable desenlace, calculando la TFG con la ecuación de Cockroft y Gault. Además, la recolección de la información no dependió del investigador sino de la información ya registrada en la base de datos.

La confusión se controló mediante un modelo de regresión multivariado, y con la exclusión de pacientes que tuvieran DM, lupus, enfermedades glomerulares, enfermedad renal aguda, reflujo vesico-ureteral y litiasis renal.
Para el análisis estadístico se utilizaron estadísticos descriptivos, estimador de asociación el $\mathrm{OR}$ con su IC: $95 \%$ y la prueba de $\mathrm{Chi}^{2}$, estableciendo como significancia estadística los valores de $p<0,05$. Se estimó un modelo de regresión múltiple para evaluar el efecto de la obesidad sobre la TFG ajustado con algunas covariables. El análisis fue realizado mediante el programa RStudio 1.3.959.

La presente investigación se clasificó en la categoría "investigación sin riesgo". Se salvaguardó la confidencialidad de la información y se respetaron los principios de bioética. Se contó con la aprobación del comité de ética de la Universidad Surcolombiana y de la ESE Carmen Emilia Ospina según acta número 020 del 2019.

\section{Resultados}

Durante el 2019 se atendieron 7.015 pacientes en el programa de riesgo cardiovascular, de los cuales 3.818 fueron excluidos por ser diabéticos, por lo que fueron analizados 3.197 pacientes con HTA que cumplieron con los criterios de inclusión.

El promedio de edad fue de $64 \pm 12,47$ años, siendo más frecuente el sexo femenino $70,58 \%(n=2.258)$. El promedio de la presión arterial sistólica y diastólica fue de $129 \pm 15,81$ y $80 \pm 9,53 \mathrm{mmHg}$, respectivamente. EI $68,95 \%(n=2.195)$ y $80,57 \%(n=2.576)$ de los usuarios tenía controlada la tensión arterial sistólica y diastólica.

Con relación al perfil lipídico, se reportó alterado el colesterol total al $26,80 \%(n=857)$, el HDL al $34,59 \%$ ( $n=1.106)$, el LDL al 7,88\% ( $n=252)$ y los triglicéridos al $40,06 \%(n=1.281)$ de los pacientes (Tabla 1$)$.

El promedio del peso fue de $71,83 \pm 16 \mathrm{~kg}$, de la talla $156,4 \pm 9,07 \mathrm{~cm}$, perímetro abdominal $96,00 \pm 11,48 \mathrm{~cm}$ y el IMC de $29,33 \pm 5,91$. El $26,11 \%(n=835)$ tenía obesidad grado I, el $9,63 \%(n=308)$ obesidad grado II y el $4,56(n=146)$ obesidad grado III.

En cuanto a las pruebas de función renal, el 2,06\% $(n=66)$ tuvo la creatinina alterada, teniendo un promedio de 0,84 $\pm 0,28 \mathrm{mg} / \mathrm{dl}$. El 6,31\% presentó alteración de la albuminuria, siendo más frecuente la microalbuminuria $(5,72 \%)$ y la proteinuria $(0,59 \%)$.

Se encontró asociación estadísticamente significativa entre la obesidad y el sexo femenino (0R:2,04; IC:95\%:1,7-2,4), la tensión arterial diastólica no con- 
Tabla 1. Creatinina, perfil lipídico y albuminuria en la población estudiada.

\begin{tabular}{|l|c|c|c|c|c|c|}
\hline & Mínima & Máxima & Mediana & Normal n (\%) & Alterada n (\%) \\
\hline Creatinina (mg/dl) & 0,3 & 5 & 0,8 & $2.810(87,89)$ & $66(2,06)$ \\
Colesterol total (mg/dl) & 75 & 467 & 167,1 & $2.106(65,87)$ & $857(26,80)$ \\
Colesterol HDL (mg/dl) & 14,8 & 183,1 & 45,1 & $1.779(55,64)$ & $1.106(34,59)$ \\
Colesterol LDL (mg/dl) & 6,7 & 369 & 111,9 & $2.571(80,41)$ & $252(7,88)$ \\
\hline Triglicéridos (mg/dl) & 32,7 & 1603,4 & 152,7 & $1.230(38,47)$ & $1.281(40,06)$ \\
\hline & Mínima & Máxima & Mediana & No albuminuria n (\%) & Microalbuminuria n (\%) & Proteinuria n (\%) \\
\hline Albuminuria (mg/24 h) & 0,5 & 1.991 & 6,4 & $1.926(60,24)$ & $183(5,72)$ & $19(0,59)$ \\
\hline
\end{tabular}

mg/dl: miligramos por decilitro.

Tabla 2. Asociación de la obesidad con el sexo, el tratamiento farmacológico, la tensión arterial y el grado de enfermedad renal crónica en población hipertensa.

\begin{tabular}{|c|c|c|c|c|c|}
\hline Variables & & $\begin{array}{c}\text { Obesidad } \\
\text { Si } \\
\text { n (\%) }\end{array}$ & $\begin{array}{c}\text { No } \\
\text { n (\%) }\end{array}$ & $\begin{array}{c}\text { OR } \\
\text { (IC:95\%) }\end{array}$ & $\mathbf{p}$ \\
\hline \multirow[t]{2}{*}{ Sexo } & Mujer & $1.011(79,11 \%)$ & $1.247(64,91 \%)$ & \multirow{2}{*}{$2,04(1,7-2,4)$} & \multirow[t]{2}{*}{$<0,01$} \\
\hline & Hombre & $267(20,89 \%)$ & $674(35,09 \%)$ & & \\
\hline \multirow[t]{2}{*}{ Tratamiento con ARA2 } & Si & $894(69.95 \%)$ & $1.223(63,66 \%)$ & \multirow{2}{*}{$1,33(1,14-1,55)$} & \multirow[t]{2}{*}{$<0,01$} \\
\hline & No & $384(30,05 \%)$ & $698(36,34 \%)$ & & \\
\hline \multirow[t]{2}{*}{ Tratamiento con IECA } & Si & $279(21,83 \%)$ & $501(26,08 \%)$ & \multirow{2}{*}{$0,79(0,67-0,93)$} & \multirow[t]{2}{*}{$<0,01$} \\
\hline & No & $999(78,17 \%)$ & $1.429(73,92 \%)$ & & \\
\hline \multirow[t]{2}{*}{ TA Sistólica } & No controlada & $407(31,85 \%)$ & $595(31,01 \%)$ & \multirow{2}{*}{$1,04(0,89-1,21)$} & \multirow[t]{2}{*}{0,6} \\
\hline & Controlada & $871(68,15 \%)$ & $1.324(68,99 \%)$ & & \\
\hline \multirow[t]{2}{*}{ TA Diastólica } & No controlada & $298(23,32 \%)$ & $323(16.84 \%)$ & \multirow{2}{*}{$1,50(1,26-1,79)$} & \multirow[t]{2}{*}{$<0,01$} \\
\hline & Controlada & $980(76,68 \%)$ & $1.596(83,16 \%)$ & & \\
\hline \multirow[t]{6}{*}{ Enfermedad Renal Crónica } & I & $769(60,10 \%)$ & $458(23,84 \%)$ & \multirow{2}{*}{$3,6(3,0-4,3)$} & \multirow[t]{2}{*}{$<0,01$} \\
\hline & II & $308(24,10 \%)$ & $661(34,40 \%)$ & & \\
\hline & IIIA & $64(05,00 \%)$ & $332(17,28 \%)$ & $8,7(6,5-11,6)$ & $<0,01$ \\
\hline & IIIB & $14(01,09 \%)$ & $225(11,71 \%)$ & $26,9(15,5-46,8)$ & $<0,01$ \\
\hline & IV & $2(00,15 \%)$ & $42(02,18 \%)$ & $36,2(8,4-146,3)$ & $<0,01$ \\
\hline & V & $121(09,46 \%)$ & $203(10,56 \%)$ & $2,8(2,1-3,6)$ & $<0,01$ \\
\hline
\end{tabular}

TA: tensión arterial.

trolada (0R:1,50; IC:95\%:1,26-1,79), la indicación de ARA2 (0R:1,33; IC:95\%:1,14-1,55), y el hecho de no tener indicado IECA (0R:0,79: IC:95\%:0,67-0,93). También se observó que a medida que progresa la enfermedad renal crónica en sus estadios, se incrementa el riesgo de tener obesidad en los pacientes que presentan hipertensión (Tabla 2). En cuanto a sus parámetros analíticos, el HDL en riesgo (0R:1,40; IC:95\%1,20-1,63) y los triglicéridos altos (0R:1,3: IC:95\%1,1-1,6) fueron más frecuentes en pacientes con obesidad. (Tabla 3).

Los pacientes obesos tuvieron una mediana de la TFG superior comparado con los no obesos (Figura 1), pero dicha diferencia solo fue observada en los estadios de ERC grado I y grado II (Figura 2). En el modelo multivariado se encontró que la obesidad, el perímetro abdo- 
Tabla 3. Asociación de la obesidad con los parámetros analíticos en población hipertensa.

\begin{tabular}{|c|c|c|c|c|c|}
\hline Parámetros analíticos & Resultado & $\begin{array}{c}\text { Obesidad } \\
\text { Si } \\
\text { n (\%) }\end{array}$ & $\begin{array}{c}\text { No } \\
\text { n (\%) }\end{array}$ & $\begin{array}{c}\text { OR } \\
\text { (IC95\%) }\end{array}$ & $\mathbf{p}$ \\
\hline Creatinina & $\begin{array}{l}\text { Alterada } \\
\text { Normal }\end{array}$ & $\begin{array}{c}21(01,82 \%) \\
1.135(98,18 \%)\end{array}$ & $\begin{array}{c}45(02,62 \%) \\
1.675(97,38 \%)\end{array}$ & $0,68(0,4-1,1)$ & 0,2 \\
\hline Albuminuria & $\begin{array}{l}\text { Micro-albuminuria } \\
\text { No albuminuria } \\
\text { Proteinuria }\end{array}$ & $\begin{array}{c}70(08,02 \%) \\
797(91,39 \%) \\
5(00,57 \%)\end{array}$ & $\begin{array}{c}113(08,99 \%) \\
1.129(89,88 \%) \\
14(01,11 \%)\end{array}$ & $\begin{array}{c}\text { Ref. } \\
0,8(0,6-1,1) \\
1,7(0,5-5,0)\end{array}$ & $\begin{array}{l}0,4 \\
0,4\end{array}$ \\
\hline Micro-albuminuria & $\begin{array}{l}\text { SI } \\
\text { NO }\end{array}$ & $\begin{array}{c}797(91,92 \%) \\
70(08,08 \%)\end{array}$ & $\begin{array}{c}1.129(90,91 \%) \\
113(09,09 \%)\end{array}$ & $1,1(0,8-1,5)$ & 0,45 \\
\hline Proteinuria & $\begin{array}{l}\text { SI } \\
\text { NO }\end{array}$ & $\begin{array}{c}797(99,37 \%) \\
5(00,63 \%)\end{array}$ & $\begin{array}{c}1.129(98,77 \%) \\
14(01,23 \%)\end{array}$ & $1,9(0,7-5,5)$ & 0,2 \\
\hline Colesterol Total & $\begin{array}{l}\text { Alto } \\
\text { Normal }\end{array}$ & $\begin{array}{l}329(27,67 \%) \\
860(72,33 \%)\end{array}$ & $\begin{array}{c}528(29,76 \%) \\
1.246(70,24 \%)\end{array}$ & $0,9(0,7-1,0)$ & 0,2 \\
\hline HDL & $\begin{array}{l}\text { Riesgo } \\
\text { Normal }\end{array}$ & $\begin{array}{l}499(43,09 \%) \\
659(56,91 \%)\end{array}$ & $\begin{array}{c}607(35,15 \%) \\
1.120(64,85 \%)\end{array}$ & $1,40(1,20-1,63)$ & $<0,01$ \\
\hline LDL & $\begin{array}{l}\text { Alto } \\
\text { Normal }\end{array}$ & $\begin{array}{c}98(08,65 \%) \\
1.035(91,35 \%)\end{array}$ & $\begin{array}{c}154(09,12 \%) \\
1.534(90,88 \%)\end{array}$ & $0,9(0,7-1,2)$ & 0,7 \\
\hline Triglicéridos & $\begin{array}{l}\text { Alto } \\
\text { Normal }\end{array}$ & $\begin{array}{l}563(55,91 \%) \\
444(44,09 \%)\end{array}$ & $\begin{array}{l}718(22,44 \%) \\
786(52,26 \%)\end{array}$ & $1,3(1,1-1,6)$ & $<0,01$ \\
\hline
\end{tabular}

n (\%) Si: muestra de pacientes con obesidad.

n (\%) No: muestra de pacientes sin obesidad.

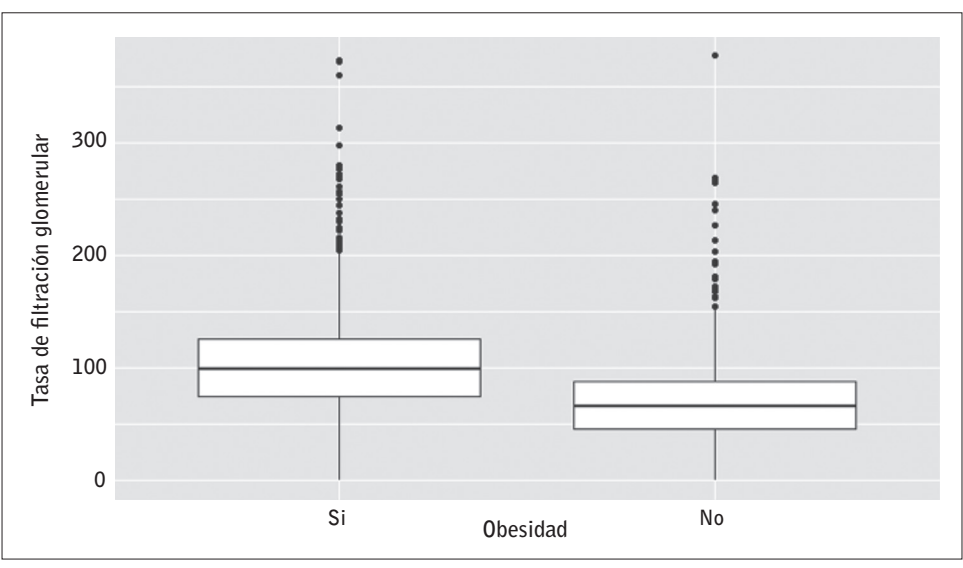

Figura 1. Mediana de la tasa de filtrado glomerular $\left(\mathrm{ml} / \mathrm{min} / 1,73 \mathrm{~m}^{2}\right)$ en pacientes con hipertensión según obesidad.

minal en riesgo, el LDL normal y la tensión arterial diastólica no controlada incrementan el promedio de la TFG en los pacientes con HTA. Cuando se realiza un análisis estratificado por clasificación de ERC, se observa que la obesidad en el estadio I y II de la ERC incrementa el promedio de la TFG, pero a partir del estadio IIIA, no se observa asociación estadísticamente significativa y a partir del IIIB los pacientes obesos presentan disminución de la TFG respecto a los no obesos (Tabla 4).

\section{Discusión}

En este estudio se encontró asociación entre la obesidad y el incremento de la TFG solo en estadios I y II. El sexo, el perímetro abdominal, la tensión arterial no controlada y el LDL fueron variables que se asociaron con la TFG, pero igualmente tienen un comportamiento diferente por grado de ERC.

La disminución de la TFG en la población a nivel mundial ha tenido un incremento exponencial en los últimos años, debido al aumento de la DM, HTA, enfermedades glomerulares $^{14,21-23}$ y a la obesidad ${ }^{14,16,17,19}$. En este estudio no se logró demostrar una menor TFG en todos los pacientes obesos, sino en aquellos con un grado mayor de 
Tabla 4. Efecto de la TFG estratificado por grado de ERC según obesidad, sexo, perímetro abdominal, tensión arterial y LDL en población hipertensa. Modelo Multivariado.

\begin{tabular}{|c|c|c|c|c|c|c|c|}
\hline Variable & $B(p)$ & ERC I & ERC II & ERC IIIA & ERC IIIB & ERC IV & ERC V \\
\hline Obesidad & $32,70(<0,01)$ & $17,70(<0,01)$ & $2,74(<0,01)$ & $1,26(0,1)$ & $-2,86(0,1)$ & $-1,12(0,7)$ & $-0,95(0,5)$ \\
\hline Sexo Masculino & $2,95(0,1)$ & $3,54(0,2)$ & $1,37(0,1)$ & $-0,01(0,9)$ & $-0,28(0,7)$ & $-3,04(<0,01)$ & $-1,34(0,4)$ \\
\hline $\begin{array}{l}\text { Perímetro } \\
\text { abdominal riesgo }\end{array}$ & $9,75(<0,01)$ & $-0,82(0,8)$ & $1,93(<0,01)$ & $-0,19(0,7)$ & $-0,35(0,6)$ & $-0,15(0,9)$ & $-0,93(0,6)$ \\
\hline $\begin{array}{l}\text { TA Sistólica } \\
\text { No controlada }\end{array}$ & $-7,53(<0,01)$ & $-5,41(0,1)$ & $0,31(0,7)$ & $-0,67(0,4)$ & $-0,11(0,9)$ & $-0,44(0,7)$ & $3,37(<0,01)$ \\
\hline $\begin{array}{l}\text { TA Diastólica } \\
\text { No controlada }\end{array}$ & $11,83(<0,01)$ & $9,14(<0,01)$ & $0,67(0,5)$ & $0,30(0,7)$ & $-0,84(0,5)$ & $-3,33(0,1)$ & $-3,89(0,1)$ \\
\hline LDL Normal & $11,03(<0,01)$ & $9,80(<0,01)$ & $-0,87(0,5)$ & $0,64(0,5)$ & $1,20(0,1)$ & $1,89(0,3)$ & $-0,07(0,9)$ \\
\hline
\end{tabular}

TA: tensión arterial; LDL: lipoproteinas de baja densidad (low density lipoproteins). B(p): coeficiente.

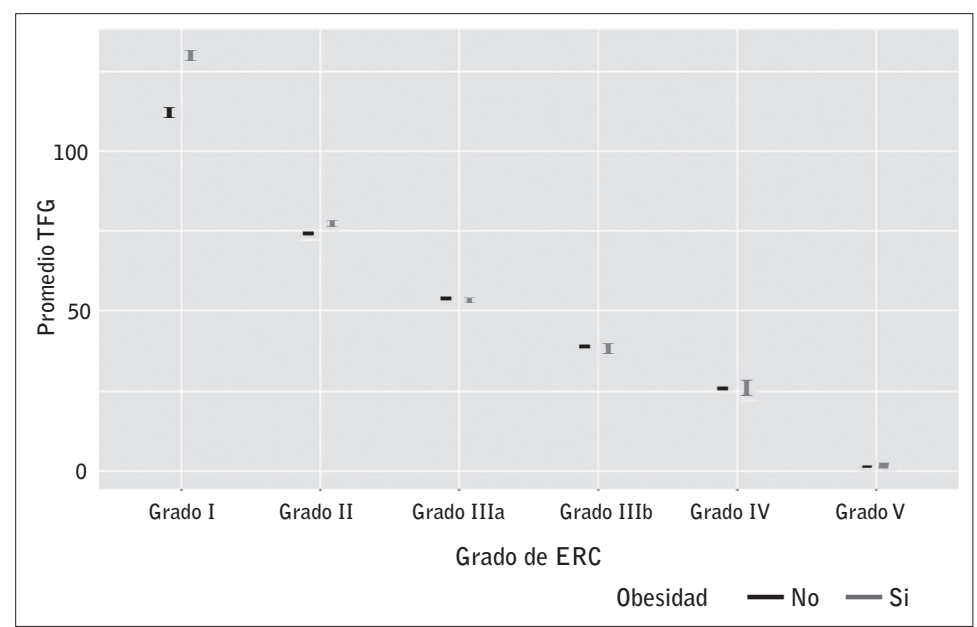

Figura 2. Promedio de la tasa de filtrado glomerular (TFG), en $\mathrm{ml} / \mathrm{min} / 1,73 \mathrm{~m}^{2}$ en pacientes con hipertensión según obesidad y grado de enfermedad renal crónica (ERC).

IIIA, y se podría deber a las múltiples causas que tiene el fallo renal. Este resultado también ha sido reportado en otros estudios ${ }^{14,16,19}$ donde encontraron que no solo los sujetos con sobrepeso y obesidad, sino también los sujetos delgados con distribución central de la grasa tienen riesgo de filtración disminuida ${ }^{24}$. Por lo tanto, el patrón central de distribución de la grasa, no el sobrepeso ni la obesidad por sí mismos, parecen ser importante para la insuficiencia renal.

En este mismo sentido, otro estudio realizado en Colombia evidenció que la ERC está condicionada por el efecto prolongado de la obesidad, junto con otros factores independientes, que actúan sobre diferentes parámetros renales funcionales que determinan el desarrollo y progresión de la enfermedad renal ${ }^{25}$. La obesidad, por sí sola, es capaz de aumentar la demanda funcional renal por un aumento de la masa corporal sin el correspondiente aumento en el número de nefronas, lo cual requiere como adaptación de un aumento del flujo plasmático renal y con él, de la velocidad de filtración glomerular. Así, independiente del desarrollo de diabetes e hipertensión, la obesidad puede provocar un síndrome de hiperfiltración glomerular explicando la existencia de microalbuminuria ${ }^{14}$.

El estudio presenta algunas limitaciones que se deben discutir. En primera medida la información proviene de revisión de historia clínica, por lo que puede haber algún sesgo en la selección de los individuos o errores de cobertura y/o calidad del dato. Por el diseño transversal, el estudio no permite establecer asociación causal entre obesidad y TFG, siendo esta la segunda limitación. No se recolectaron algunas variables asociadas a la TFG, tales como, tiempo de la obesidad, los estilos de vida de los usuarios, la etnia, el nivel educativo, exámenes clínicos como la hemoglobina. La cuarta limitación es que la clasificación de ERC se realizó a partir de los registros de la historia clínica y no de un diagnóstico médico, por lo que podría haber pacientes con mala clasificación. A pesar de estas limitaciones, el estudio contempló un tamaño de muestra significativo para la población y mediante un modelo de regresión pudo demostrar el aporte de un conjunto de variables para la TFG.

Referente a las implicaciones para la práctica de Enfermería, con los resultados del estudio se evidencia la importancia de la educación para salud acerca de las consecuencias nocivas de la obesidad y su asociación con la enfermedad renal, abogando por una estilo de vida salu- 
dable y la implementación de políticas públicas de salud que promuevan medidas preventivas alcanzables ${ }^{26}$. En este sentido, se resalta el liderazgo de los profesionales de enfermería en los procesos educativos sobre autocuidado, dentro de los programas de atención primaria en salud y gestión del riesgo, que permitan el alcance de las metas terapéuticas de peso, perímetro abdominal y perfil lipídico en pacientes con hipertensión arterial.

Se concluye que se encontró asociación entre obesidad e incremento de la TFG solo en estadios de ERC I y II. EI perímetro abdominal, la tensión arterial no controlada y el LDL fueron variables que influyen en el promedio de la TFG, pero igualmente tienen un comportamiento diferente por grado de ERC.

Recepción: 03-10-20

Aceptación: 10-01-21

Publicación: 30-03-21

\section{Bibliografía}

1. Villena Chávez JE. Prevalencia de sobrepeso y obesidad en el Perú. Rev. peru. ginecol. obstet. 2017;63(4):593-8.

2. Barrera Dussán N, Ramos-Castañeda JA, Barrera Dussán N, Ramos-Castañeda JA. Prevalence of malnutrition in children under 5 years of age. Comparison between OMS parameters and their adaptation to Colombia. Univ Salud. 2020;22(1):91-5.

3. Organización Mundial de la Salud (OMS). Obesidad y sobrepeso. [consultado 29 jun 2020]. Disponible en: https://www.who.int/es/news-room/fact-sheets/ detail/obesity-and-overweight.

4. Moreno GM. Definición y clasificación de la obesidad. Rev. med. Clín. Las Condes. 2012;23(2):1248.

5. Cardona MS. ENSE Encuesta Nacional de Salud España 2017. 2017:37.
6. Barrera-Dussán N, Fierro-Parra EP, Puentes-Fierro LY, Ramos-Castañeda JA. Prevalence and social determinants of malnutrition in children under 5 years affiliated to the SISBEN of the urban area of the municipality of Palermo in Colombia, 2017. Univ Salud. 2018;20(3):236-46.

7. Aranceta-Bartrina J, Pérez-Rodrigo C, Alberdi-Aresti G, Ramos-Carrera N, Lázaro-Masedo S. Prevalencia de obesidad general y obesidad abdominal en la población adulta española (25-64 años) 2014-2015: estudio ENPE. Rev Esp Cardiol. 2016;69(6):579-87.

8. Martín FB, Riscos MÁG, Martín JL, Junco LS, Macías RG, Delgado RM, et al. Obesidad y supervivencia a los 5 años en pacientes con insuficiencia renal crónica avanzada. Enferm Nefrol 2007;10(3):2348.

9. Marqueta de Salas M, Martín-Ramiro JJ, Rodríguez Gómez L, Enjuto Martínez D, Juárez Soto JJ. Hábitos alimentarios y actividad física en relación con el sobrepeso y la obesidad en España. Rev. esp. nutr. hum. diet. 2016;20(3):224-35.

10. Ramírez JC, Rojas LA, Romero OC, Reynoso J. Análisis situacional de la prevalencia e incidencia de la obesidad en Latinoamérica: caso México y Colombia. Educación y Salud Boletín Científico Instituto de Ciencias de la Salud Universidad Autónoma del Estado de Hidalgo. 2017;6(11).

11. Chescheir N. Obesidad en el Mundo y su Efecto en la Salud de la Mujer. Obstet Gynecol. 2011;117(1):1213-22.

12. Barrera-Cruz A, Rodríguez-González A, Molina-Ayala MA. Escenario actual de la obesidad en México. Rev. Med. Inst. Mex. Seguro Soc. 2013;51(3):292-9.

13. Gutiérrez-Rodríguez D. Comportamiento del indice de masa corporal en la enfermedad renal cronica en pacientes con hipertensión arterial. Revista Electrónica de Portales Médicos [Internet] 2007 Mayo [consultado 27 feb 2021]. Disponible en: https://www.portalesmedicos.com/publicaciones/ articles/524/1/Comportamiento-del-indice-de-masa-corporal-en-la-enfermedad-renal-cronica-en-pacientes-con-hipertension-arterial. 
14. Navarro G, Ardiles L. Obesidad y enfermedad renal crónica: Una peligrosa asociación. Rev Med Chile 2015;143(1):77-84.

15. Álvarez SD. Obesidad y riñón. Rev Cuba Pediatr 2019;91(1).

16. Silvariño $R$, Gadola $L$, Ríos P. Obesidad y enfermedad renal crónica. Rev. Urug. Med. Int. 2017;2(3):3-23.

17. Kovesdy CP., Furth S., Zoccali C. Obesidad y enfermedad renal: consecuencias ocultas de la epidemia. Nefrología 2017;37(4):360-9.

18. Otero González A, De Francisco ALM, Gayoso $P$, Garcia López F. Obesidad y funcion renal. datos del estudio epidemiologico: Prevalencia de la enfermedad renal cronica en España. Estudio EPIRCE. Nefrología 2018;38(1):107-8.

19. Ramírez NJ, De La Cruz de Vieytez MS, Rivas M. Obesidad: factor de riesgo de enfermedad renal crónica. Anuario de investigación 2016;5:377-86.

20. Calculadora de nefrología y fórmulas. Sociedad Española de Nefrología. Madrid. [consultado 1 sep 2020]. Disponible en: https://www.senefro.org/modules.php? name $=$ nefrocalc.

21. González BS, Pascual MR, Guijarro LR, González AF, Puertolas OC, Latre LMR. Enfermedad renal crónica en Atención Primaria: prevalencia y factores de riesgo asociados. Aten Primaria 2015;47(4):236-45.
22. Gorostidi M, Sánchez-Martínez $M$, Ruilope LM, Graciani A, Juan J, Santamaría R et al. Prevalencia de enfermedad renal crónica en España: impacto de la acumulación de factores de riesgo cardiovascular. Nefrología 2018;38(6):606-15.

23. Pascual V, Serrano A, Pedro-Botet J, Ascaso J, Barrios V, Millán J et al. Enfermedad renal crónica y dislipidemia. Clin Investig Arterioscler 2017;29(1):22-35.

24. Pérez Miguelsanz MJ. Distribución regional de la grasa corporal. Uso de técnicas de imagen como herramienta de diagnóstico nutricional. Nutr Hosp 2010;(2):207-23.

25. Parodi LC, Jiménez EN, Quiroz YA, Avendaño AL, Varela VM, Torres HJG, et al. Asociación de obesidad con la enfermedad renal crónica de pacientes atendidos en la Clínica de la Costa. 2005-2014. Rev. Colomb. Nefrol. 2016;3(1):14-9.

26. Huaman-Carhuas L, Bolaños-Sotomayor N. Sobrepeso, obesidad y actividad física en estudiantes de enfermería pregrado de una universidad privada. Enferm Nefrol 2020;23(2):184-90.

Este artículo se distribuye bajo una Licencia Creative Commons Atribución-NoComercial 4.0 Internacional. https://creativecommons.org/licenses/by-nc/4.0/ 